\title{
Evaluation of Liposomal and Microbubbles Mediated Delivery of Doxorubicin in Two-Dimensional (2D) and Three-Dimensional (3D) Models for Breast Cancer
}

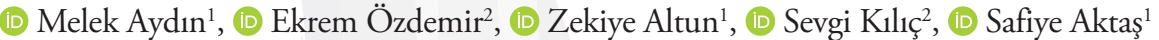 \\ ${ }^{1}$ Department of Basic Oncology, Institute of Oncology, Dokuz Eylül University, İzmir, Turkey \\ ${ }^{2}$ Department of Chemical Engineering, İzmir Institute of Technology, İzmir, Turkey
}

\begin{abstract}
Objective: Liposomal cancer treatment strategies are useful in removing the side effects that were the main concern in recent years. In this study, we prepared microbubble (MBs) conjugated with DOX-loaded liposomes (DOX-loaded MBs) and investigated their effectiveness in in vitro breast cancer cells in two dimensions (2D) and three dimensions (3D).

Materials and Methods: With this aim, breast cancer cells with different features (4T1, MDA-MB231, MCF-7) were growth in 2D and 3D dimensions. The cytotoxic and cell death effects under different conditions, durations and doses were evaluated with WST-1, trypan-blue, colony counts. Apoptotic effects were investigated with flow cytometric Annexin-V-PI and immunohistochemical (Ki-67, caspase 3, 8, 9) methods.

Results: After free DOX and LipoDOX were applied, the proliferation index of three cell lines reduced. Intrinsic and extrinsic apoptotic pathways were activated in both 2D and 3D models. However, this effect was observed at lower levels in the 3D model due to the difficulty of diffusion of DOX into the spheroids. Additionally, the suitability of the 3D model for breast cancer cells was supported by formation of ductus-like structures and spheroids. Cell deaths were not observed significantly with the DOX-loaded microbubbles due to rising of MBs to the surface and not reaching spheroids held in matrigel of $3 \mathrm{D}$ model.

Conclusion: DOX and LipoDOX showed anti-proliferative and apoptosis-inducing effects in breast cancer cells. However, these effects indicated variability depending on the cell lines and 2D or 3D model types.
\end{abstract}

Keywords: $3 \mathrm{D}$ cell culture, breast cancer, doxorubicin, liposome, microbubble

Cite this article as: Aydın M, Özdemir E, Altun Z, Kılıç S, Aktaş S. Evaluation of Liposomal and Microbubbles Mediated Delivery of Doxorubicin in Two-Dimensional (2D) and Three-Dimensional (3D) Models for Breast Cancer. Eur J Breast Health 2021; 17(3): 274-282

\section{Key Points}

- Doxorubicin can be delivered with liposome and microbubbles.

- $\quad 3 \mathrm{D}$ spheroid model is suitable for in vitro breast cancer studies.

- $\quad 3 \mathrm{D}$ in vitro models are difficult to test microbubble loaded drugs.

\section{Introduction}

Cancer is one of the leading causes of deaths worldwide. In women, breast cancer is the most commonly diagnosed cancer. In fact, it is the second most common cause of cancer death among women $(1,2)$. In 2020, breast cancer accounted for $-684,000$ deaths worldwide according to GLOBACON (https://doi.org/10.3322/caac.21660). Several studies are being conducted to devise new strategies to overcome or reduce the common side effects associated with the administration of chemotherapeutics $(3,4)$. One of the strategies that is being widely explored involves the use liposomes to deliver entrapped cancer drugs. Commercially approved liposomal formulations include lipoplatin (cisplatin encapsulated in liposome) and LipoDOX [doxorubicin (DOX) entrapped in liposome] (5-7). Currently, the therapeutic safety and efficacy of LipoDOX for the treatment of triple-negative breast cancer is being evaluated in clinical trials and experimental studies in different combinations (8, 9). Most of the current research is focused using of poly (ADP-ribose) polymerase (PARP) inhibitors (10), in modified tandem peptide form (11), with liposomal immunotherapy (12), and in local gel form (13).

Microbubbles (MB), small gas-filled bubbles with size 0.5-10 $\mu \mathrm{m}$, find wide applications in industry, medicine, and life sciences. Generally, microbubble shell structure is characterized by the presence of phospholipid with polyethylene glycol (PEG) as emulsifying agent. When 
ultrasound is applied to $\mathrm{MBs}$, the high pressure induces a compression movement resulting in the reduction in the size of MBs. Further, the reduction in pressure is accompanied by an increase in the size of MBs (14). Thus, the ultrasound images generated from the signals obtained from oscillations of microbubbles provide high quality and clearly comprehensible information. All theses features make MBs a promising contrast agent for ultrasound imaging. During ultrasound imaging, the oscillation of MBs results in the development of transient pores in the cell walls. As the intensity of ultrasound increases, MBs form a jet flow. This results in the opening of holes in cell walls, allowing the flow of chemotherapeutic drug into the cells (15). Thus, MBs act as excellent drug delivery agents.

In the present study, the suitability of MBs as delivery agents for DOX was explored. Doxorubicin-loaded MBs were developed by attachment of DOX-loaded liposomes to ultrasound contrast agent microbubbles via avidin-biotin bridge. The study aimed to evaluate and compare the therapeutic of efficacy of DOX-loaded MBs and DOX-loaded liposomes with free DOX in in vitro two-dimensional (2D) and threedimensional (3D) models for breast cancer.

\section{Material and Methods}

\section{Chemicals}

Clinical grade liposomal doxorubicin (Caelyx LipoDOX) was purchased from Janssen Pharmaceutica NV, Belgium. Doxorubicin $\mathrm{HCl}$ (Adriamycin) India, was procured from West-Ward, Franco Indian Pharmaceuticals. Distearoyl glycero phosphocholine (DSPC, 99\%), 1,2 distearoylsnglycerol 3 phosphoethanolamine N [methoxy(polyethylene glycol)2000] (DSPE-PEG2000), and biotinylated DSPE-PEG2000 were obtained from Avanti Polar Lipids (Alabaster AL). Cholesterol and polyoxyethylene-40-stearate (PEG40St) were purchased from Sigma Aldrich (St. Louis, MO).

\section{Preparation and characterization of microbubbles, DOX-loaded} liposomes (LipoDOX), and DOX-loaded MBs

Microbubbles were prepared using DSPC as lipid, (PEG40St) as emulsifier, and biotinylated DSPE-PEG2000. MBs were prepared using the process described previously by Onercan (16). Since Caelyx LipoDOX is not suitable for conjugation with MBs, LipoDOX was prepared in-house using a mixture of DSPC, cholesterol, DSPE-PEG, and biotinylated DSPE-PEG as described before (16). In order to prepare DOX-loaded MBs, preformed LipoDOX and MBs in same $\% 50$ weights were mixed and incubated for $15 \mathrm{~min}$ on a rotary mixer at room temperature. Unbound LipoDOX was removed from the solution by flotation technique. Unbound Lipodox was at bottom and MB loaded Lipodox was flotaning. The amount of DOX bound to the MBs was determined using a fluorescence spectrophotometer (SHIMADZU) with emission signal at $595 \mathrm{~nm}$ upon excitation with a $470 \mathrm{~nm}$ laser. Characteristics for the various formulations used in the study are summarized in Table 1.

\section{Formation of three-dimensional (3D) cell cultures and spherule diameter measurement}

MCF-7 (HTB-22, ATCC) (hormone receptor positive human cell line), MDA-MB-231 (HTB-26, ATCC) (triple negative human cell line expressing human epidermal growth factor receptor 2 (HER2), non-amplified), and 4T1 (CRL-2539, ATCC) (triple negative mouse cell line) breast cancer cell lines were used in the study. The cells were cultivated using Dulbecco's Modified Eagle Medium, DMEM (Corning, 10-017 CV) for MDMA-MB-231 and RPMI-1640 medium (Corning, 15-040 CV) for 4T1 and MCF7 supplemented with 1\% L-glutamine (Corning, 25-005 CI), penicillin/streptomycin (Corning, 30-002-CI), and 10\% fetal bovine serum (Corning, 35-010-CV). The cell lines were maintained at $37^{\circ} \mathrm{C}$ in a humidified incubator under $5 \% \mathrm{CO}_{2}$. All the experiments were repeated thrice, at minimum three different time points.

For 3D cell culture, a Matrigel basement membrane matrix (Corning) was first brought to liquid state by incubation at $4{ }^{\circ} \mathrm{C}$. The material was swirled to ensure proper dispersion of the material. In a 96 well plate, 1000 cells per well were seeded with Matrigel in 96 well plate, 6 wells per condition (17-19). The cells were incubated at $37{ }^{\circ} \mathrm{C}$ in a humidified incubator at $5 \% \mathrm{CO}_{2}$ for 24 hours. After 24 hours, the cells were treated with optimized doses of DOX, LipoDOX, and DOX-loaded MBs. For each treatment six wells were used. Samples were further incubated at $37{ }^{\circ} \mathrm{C}$ in a humidified incubator at $5 \% \mathrm{CO}_{2}$ for 48 hours. Post 48 hours, an inverted light microscope (LEICA DMIL)) was used to image and count the number and diameter of spherules formed in each well. The cells were further stained with trypan blue to evaluate the number of live and dead cells. For trypan blue staining, $10 \mathrm{~mL}$ of the dye was added per 96 wells, media was removed, and number of blue and transparent cells were counted using a microscope. In addition to this, the cells seeded in $25 \mathrm{~cm}^{2}$ flasks were treated with effective dose of DOX, LipoDOX, and DOXloaded MBs for appropriate time and exposed to ultrasound. The cells without any treatment were used as controls for comparison. For immunohistochemical analysis, colonies were collected using a cell scraper and paraffin blocks were prepared. Immunohistochemical analysis was performed on the sections in $3 \mathrm{D}$ to evaluate the expression of Ki-67, caspase-3, caspase-8, and caspase-9.

This study was approved by Dokuz Eylül University, Noninvasive Ethics Committee (date: 09.11.2020, no: 2017/26-22).

\section{Flow pattern}

Breast cancer cells were grown on the surface of sterile slides inserted in a flask (4-well Multidish, Nunc). For 2D model, cells were directly seeded onto the slides at a cell density of $10,000 \mathrm{cell} / \mathrm{ml}$. For in vitro

Table 1. Characterization results for various formulations used in the study

\begin{tabular}{|c|c|c|c|c|}
\hline Sample & DOX concentration $(\mathrm{mg} / \mathrm{ml})$ & Sample & Concentration (MB/ml) & Mean size $(\mathrm{mm})$ \\
\hline LipoDOX & 493.8 & $\begin{array}{l}\text { MB before } \\
\text { conjugation }\end{array}$ & $2.66 \times 10^{9}$ & 3.11 \\
\hline DOX-loaded MBs & 15.4 & $\begin{array}{c}\text { MB after } \\
\text { conjugation }\end{array}$ & $7.90 \times 10^{8}$ & 2.99 \\
\hline
\end{tabular}


3D studies, $21.8 \mathrm{~cm}^{2} /$ well area was seeded with $10^{6}$ cells mixed in 1 $\mathrm{mL}$ of cold liquid Matrigel per unit surface. The cells were incubated at $37^{\circ} \mathrm{C}$ to allow hardening of the Matrigel for 30 minutes. Further, complete medium was added and plate was incubated for 48 hours for spherule formation. After 48 hours, the cells were used for flow experiments. A special flow chamber was designed to perform the experiments in 2D and 3D. The cells were placed in the flow chamber and the flow experiments were performed. The MB loaded DOX were fluxed through the flowing apparatus over cells.

\section{Measurement of cell proliferation using WST-1 assay}

In a 96 well plate, the cells were seeded at a density of $10^{4}$ cells/well and treated with different doses of DOX and LipoDOX (0.5, 1.5, $2.5,5,10$, and $20 \mu \mathrm{M})$. For each treatment six wells were used. Cell proliferation assay was performed after 24 and 48 hours of incubation using WST-1 solution (Roche, 11644807001). To evaluate the effect of dose and time on cell viability, the cells were treated with WST1 at a concentration $10 \mu \mathrm{L} /$ well and incubated at $37{ }^{\circ} \mathrm{C}$ for 1 hour. Cell proliferation was measured in terms of absorbance at $450 \mathrm{~nm}$ using an ELISA reader (Thermo). LD50 (50\% Lethal dose) for DOX and LipoDOX on these breast cancer cell lines was calculated as [(absorbance of test sample - absorbance of control)/control].

\section{Flow cytometry-based measurement of apoptosis and necroptosis using Annexin-V-FITC/PI staining}

Post the treatment of cells with different formulations, treated and control cells were collected and centrifuged at 1,200 rpm for $5 \mathrm{~min}$. The supernatant was carefully removed without disturbing the cell pellet. The resulting cell pellet was resuspended in $100 \mu \mathrm{L}$ of PBS buffer. For FACS analysis, one tube for each sample without staining was used for gating. The remaining tubes were stained with propidium iodine (PI) alone, FITC-Annexin-V alone or combination of PI and Annexin-V (Sigma-Aldrich A9210). All the tubes were incubated for 15 minutes at 4 centrigrate degree. After 15 minutes of incubation, binding buffer was added to each tube and flow cytometry analysis (BD-Accuri, C6 Flow Cytometer) was performed (FITC with FL 1 dedector at 530/30 $\mathrm{nm}$ filter and PI with FL2 dedector at 585/42 nm). After appropriate gating, early apoptotic and late apoptotic/necrotic cells were identified using Annexin-V staining alone and a combination of Annexin- $\mathrm{V}$ and PI, respectively. The data for flow cytometry analysis was expressed as percentage calculated in comparison to the initial cell counts.

\section{TUNEL and immunohistochemistry}

For each cell line, cells were seeded in a $25 \mathrm{cc}$ flask and treated with LC50 doses (obtained in the previous assay) for 24 or 48 hours (time and dose for each treatment are given in results section). The cells were collected in $15 \mathrm{~mL}$ tubes and centrifuged at rpm. The supernatant was carefully removed and the cells were fixed by the addition of methanol. After 24 hours, the cells were blocked for tissue monitoring and paraffin blocking was performed. Sections of $5 \mu \mathrm{m}$ thickness were placed on positive-loaded slides.

The sections were incubated in an oven at $60{ }^{\circ} \mathrm{C}$ overnight. Further, the sections were deparaffinized with water and $\mathrm{TdT}$ labeling was performed using Biotin d-UTP kit (Genscript L00290) according to the manufacturer's protocol. The resulting sections were further stained with diaminobenzidine, while background staining was performed using hematoxylin. In order to calculate $\%$ apoptosis, the number of brown positive cells were counted per 5,000 cells and normalized for total cell count. The identification of apoptotic cells was performed on
For immunohistochemistry analysis, the samples were treated with primary antibody for Ki-67 (Elabscience, e-pp-24636), caspase-3 (Bioss, Bs-0081R), caspase-8 (Bioss, Bs-0052R), and caspase-9 (Bioss, Bs-0049R) for 30 minutes. This was followed by treatment with anti-rabbit haptoglobin related protein secondary antibody at 1:200 dilution in automatic immunohistochemistry staining device (Ventana Discovery). The treatment with diaminobenzidine resulted in dark brown color. During analysis, positivity was used as the sole criteria, while sample intensity was not considered. Number of positive and negative cells were counted and percentage was calculated. For background staining hematoxylin dye was used. All sections showed positive cell counts, and cells and colonies were imaged using a light microscope (Olympus, Germany).

\section{Statistical analysis}

Data are represented as mean \pm standard deviation. Statistical analysis was performed using SPSS 22.0 program. Nonparametric tests were used for analysis and the results of the study were considered statistically significant for $\mathrm{p}<0.05$.

\section{Results}

\section{Cell proliferation assay using WST-1}

The effect of DOX dose and duration of treatment on cell viability was evaluated in three breast cancer cell lines using WST-1. Each cell line was treated with six different concentrations of DOX $(0.5,1.5$, 2.5, 5, 10, and $20 \mu \mathrm{g} / \mathrm{mL}$ ) for 24 and 48 hours. As shown in Figure 1, the effective near LC50 dose for 4T1, MCF-7, and MDA-MB-231 cells was found to be $1.5,1.5$, and $3 \mu \mathrm{g} / \mathrm{ml}$, respectively. Both $4 \mathrm{~T} 1$ and MDA-MB-231 cell lines showed LC50 time of 48 hours, while MCF7 was characterized by LC50 time of 24 hours (Figure 1).

To evaluate the effect of LipoDOX on cell viability, all three cell lines were treated with seven different doses of LipoDOX $(0.1,0.25,0.5$, $0.75,1,1.5$, and $2 \mu \mathrm{g} / \mathrm{ml}$ ) and LC50 time and dose were calculated. The effective near LC50 time and dose for 4T1 cells was 48 hours and $1.5 \mu \mathrm{g} / \mathrm{ml}$, respectively. In comparison to this, MCF-7 and MDAMB-231 showed lower LC50 doses of $0.75 \mu \mathrm{g} / \mathrm{ml}$ and $0.25 \mu \mathrm{g} / \mathrm{ml}$, respectively, which were observed at LC50 time of 24 and 48 hours, respectively (Figure 2 ).

\section{Assessment of spherule number and diameter in 3D matrigel model}

To assess the number and diameter of spherules formed, the cells were grown in 3D Matrigel and treated with optimized doses of DOX, LipoDOX, and DOX-loaded MBs. After 48 hours of treatment, spherule formation was evaluated using light microscopy. Spheroids displayed significant variability in terms of size, diameter, and numbers. In the control group, large size spheroids with ductus structures were observed. Interestingly, spheroids formed in case of MDA-MB-231 cell were smaller in size as compared to the spheroids for MCF-7. In comparison to these, most successful and dense spheroid formation was observed in $4 \mathrm{~T} 1$ cells, where large of number of spheroids were formed. The treatment of all the cell lines with DOX resulted in significant decrease in spheroid diameters as compared to the control cells. These DOX treated spheroids showed significant necrosis and apoptosis. In case of DOX treated MCF-7 cells, spheroids were preserved, however, they were smaller size. A lower apoptosis rate was observed in MCF-7 cells as compared to other cell lines, which was in agreement with the aforementioned observation. In comparison to this, DOX treatment in MDA-MB-231 cells resulted in fewer number 
of spheroids that showed apoptotic morphology. For all three cell lines included in the study, spherule counts and diameter were significantly reduced post administration of DOX and LipoDOX. Mean values for number and diameter of spherules observed for different treatment groups are summarized in Table 2. Figure 3 shows microscopic images of the 3D spherules formed for three breast cancer cell lines.
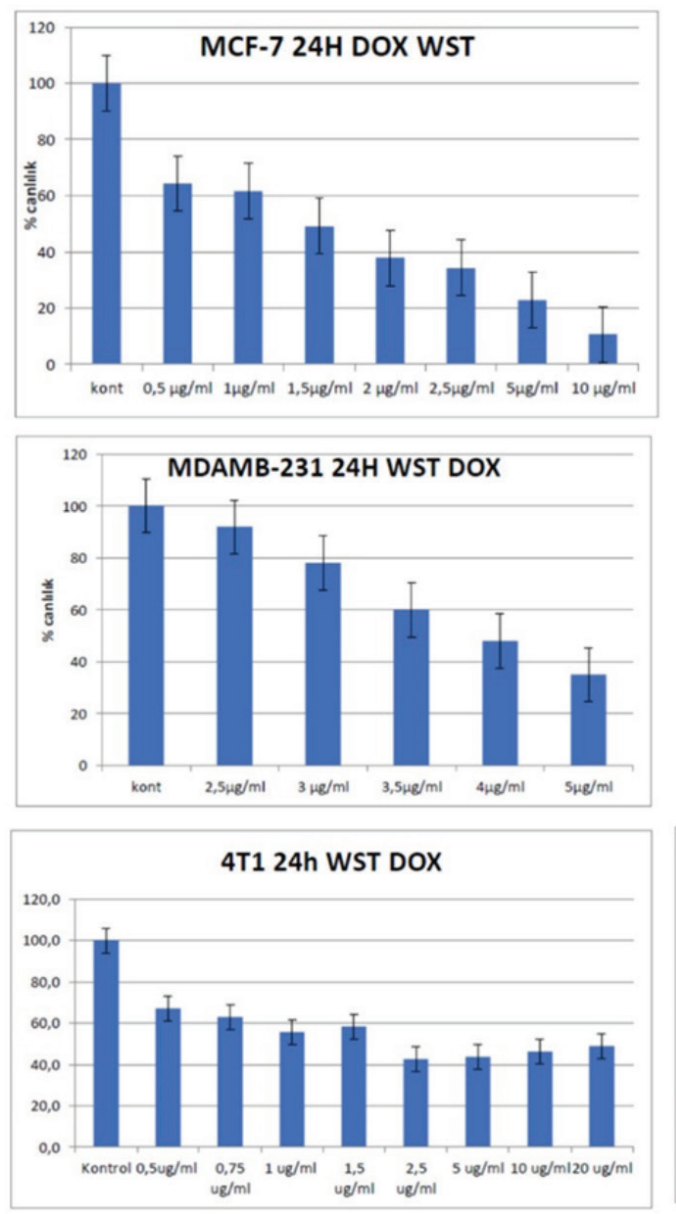

Aydın et al. Liposomal Doxorubicine in Breast Cancer Cells

\section{Technical Issues associated with 3D model studies}

After successful development of 3D culture models for three cell lines, paraffin blocks were prepared and spheroid morphologies were assessed using hematoxylin-eosin staining. In Matrigel matrix (Corning, 354234), the cells formed a specific structure around the spheroids which was similar to connective tissue. The occasional occurrence of
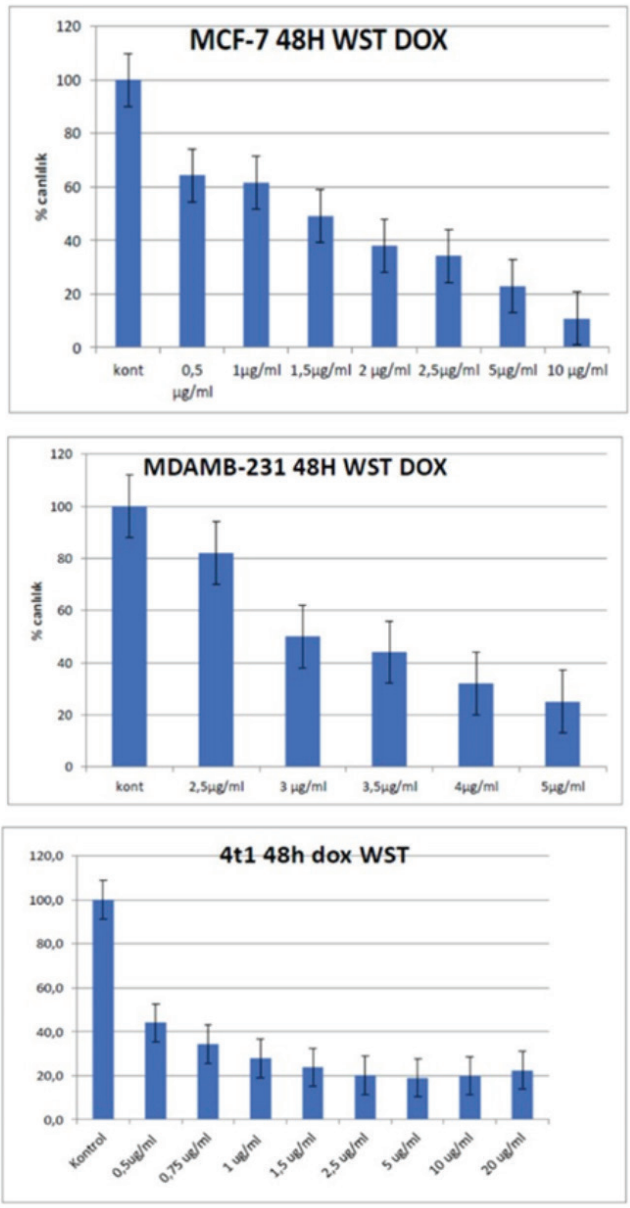

Figure 1. Doxorubicin WST-1 viability percentages compared with control in breast cancer cell lines. The effective near LD50 time and dose for $4 \mathrm{~T} 1$ cells was 48 hours and $1.5 \mu \mathrm{g} / \mathrm{ml}, 24$ hours and $1.5 \mu \mathrm{g} / \mathrm{ml}$ for MCF7 cells, 48 hours and $3 \mu \mathrm{g} / \mathrm{ml}$ for MDAMB-231 cells ( $p<0.05$ ).

Table 2. Mean spherule number and diameters obtained in 3D cell culture model for three cancer cell lines. Mean spherule number and diameter were found to be reduced in DOX and LipoDOX treated groups as compared to control group, for all three cell lines $(p<0.05)$

\begin{tabular}{lcccc}
\hline 3D Matrigel model & Mean spherule number & Standard derivation & Mean spherule diameter ( $\boldsymbol{\mu m}$ ) & Standard derivation \\
\hline 4T1 control & 302 & \pm 18 & 306.2 & \pm 80.4 \\
4T1 DOX & 43 & \pm 8 & 104.4 & \pm 95.5 \\
4T1 LipoDOX & 82 & \pm 13 & 152.2 & \pm 46.2 \\
MCF-7 control & 338 & \pm 21 & 336.3 & \pm 90.1 \\
MCF-7 DOX & 182 & \pm 36 & 184.01 & \pm 50.8 \\
MCF-7 LipoDOX & 118 & \pm 42 & 227.04 & \pm 67.1 \\
MDA-MB-231 control & 286 & \pm 28 & 280.06 & \pm 120.8 \\
MDA-MB-231 DOX & 27 & \pm 7 & 76.21 & \pm 20.7 \\
MDA-MB-231 LipoDOX & 46 & \pm 15 & 117.01 & \pm 71.2 \\
\hline LipoDOX: Doxorubicin (DOX) entrapped in liposome; MB: Microbubbles & &
\end{tabular}


apoptotic objects in the center of the spheroids and ductus structures was quite significant, particularly in MCF-7. These observations provided evidence for the suitability of the structures formed in $3 \mathrm{D}$ cultures for breast cancer studies. The ductus structures were found to be located in the center of the spheroids.

In $4 \mathrm{~T} 1$ cells, the number of spheroids were higher as compared to other two cell lines. The treatment of cells with DOX resulted in a reduction in spheroid diameters and cell counts per spheroid. However, the cell viability was found to be higher in $4 \mathrm{~T} 1$ cells as compared to other cells in 3D model. Post DOX treatment, 4T1 cells in 3D model were characterized by disrupted and reduced ductus structures. All three cell lines exhibited high proliferation index in 3D cell cultures. However, the identification of $3 \mathrm{D}$ model proliferation index was difficult as compared to $2 \mathrm{D}$ cultures.

The treatment of cells with DOX-loaded MBs failed to produce any results. This was mainly attributed by rising of MBs to the surface that remained under the Matrigel and did not diffuse owing to their large size. DOX dissolved in the medium could easily diffuse through the pores of the Matrigel and bound to the cells; however, the spheroids formed above the Matrigel did not allow the transition of liposomes
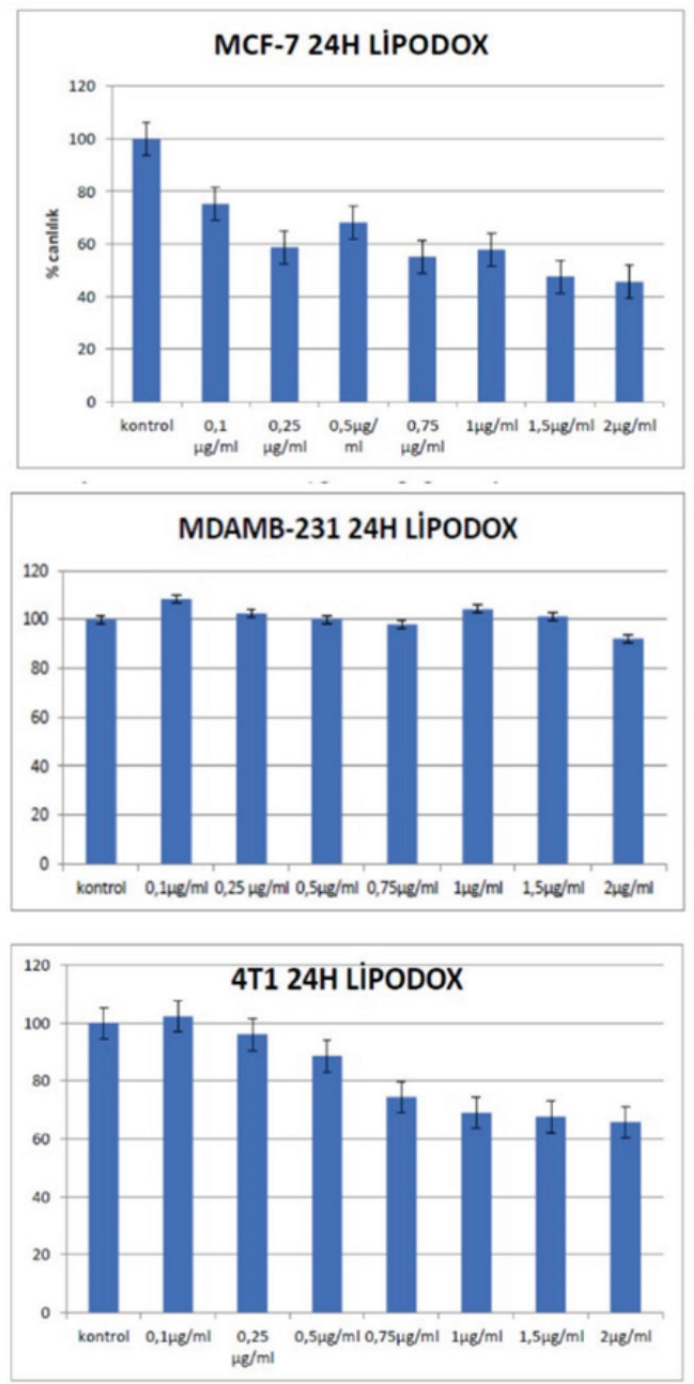

and $\mathrm{MBs}$ into the pores. As a result, DOX-loaded MBs did not pass through the pores and failed to reach the cells. Therefore, DOX-loaded MBs were not included in the following proliferation and apoptosis experiments.

For all three cell lines, the decrease in mean spherule number and diameter upon treatment with DOX and LipoDOX was found to be statistically significant as studied using Mann-Whitney $U$ test $(\mathrm{p}<0.05)$.

\section{Flow pattern findings}

The flow experiments were successfully performed in 2D model; however, 3D model could not be used for flow pattern studies owing to the inability of the drug to enter the spherules. DOX and LipoDOX were successfully applied to the cells in 2D model. A model to perform shear stress formulation was adapted. Results for the same will be provided in our future studies. One of the aims of the flow experiments was to utilize ultrasound to burst the MBs near the cells. This would have allowed the release of DOX from the liposomes, while the formation of transient pores in the cells by sonoporation would have assisted in the passage of the drug molecules. However, the experiment could not be performed as the MBs added to the
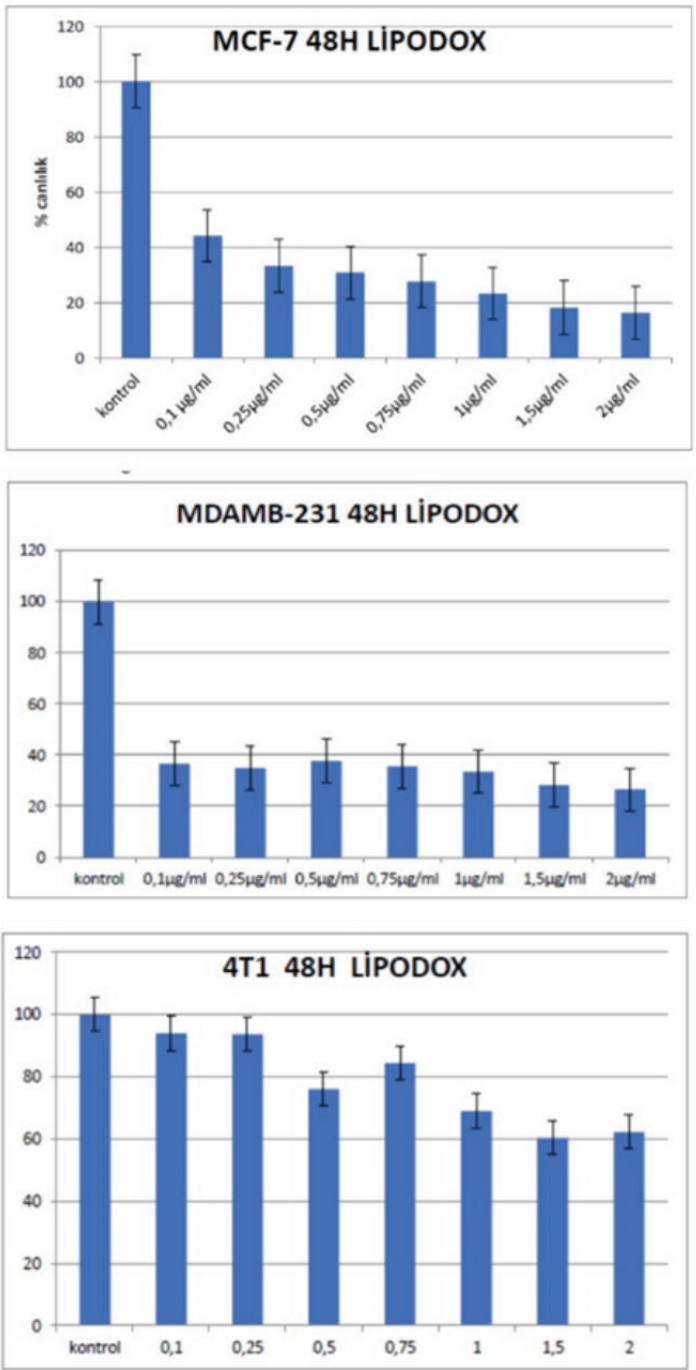

Figure 2. Liposomal-Doxorubicin WST-1 viability percentage compared with control in breast cancer cell lines. The effective near LD50 time and dose as 48 hours and $1.5 \mu \mathrm{g} / \mathrm{ml}$ for $4 \mathrm{~T} 1$ cells, 24 hours and $0.75 \mu \mathrm{g} / \mathrm{ml}$ for MCF7 cells, 48 hours and $0.25 \mu \mathrm{g} / \mathrm{ml}$ for MDAMB-231 cells $(p<0.05)$. 
medium for ultrasound destruction floated. As a result, these MBs did not contact the cells on the surface and failed to show any significant binding.

\section{Apoptosis and necroptosis findings with flow cytometric Annexin- V-FITC/PI staining}

The cells were treated with DOX and LipoDOX using the effective LC50 dose and time optimized in the previous experiments. Post treatment, the cells were collected, stained with Annexin-V-FITC/ $\mathrm{PI}$, and assessed using flow cytometry. The treatment of the three breast cancer cells with DOX and LipoDOX using optimized dose and incubation time resulted in the induction of cell death via necrosis (Table 3). For DOX treatment, the necrosis rate was found to be $95.5 \%-99.1 \%$, while LipoDOX showed necrosis rate of $75.8 \%$ 87.4\%. Mann-Whitney U analysis displayed statistically significant difference among control, DOX, and LIPODOX groups for all the cell lines $(\mathrm{p}<0.05)$.

\section{TUNEL and immunohistochemistry findings}

In 3D model studies, terminal deoxynucleotidyl transferase dUTP nick end labeling (TUNEL) staining was used for the identification

\section{Aydın et al. Liposomal Doxorubicine in Breast Cancer Cells}

of apoptosis in the cells. For 3D model of $4 \mathrm{~T} 1$ cells, 3\% and 35\% apoptosis was observed in the control and DOX treated groups, respectively (Table 4). Similar increase in apoptosis was observed for MCF-7 cells with DOX treatment, where apoptosis of 5\% and $25 \%$ was observed in the control and DOX treated cells, respectively. In case of MDA-MB-231 cells, DOX treatment resulted in an increase in apoptosis to $24 \%$. The treatment of the cells with LipoDOX was also accompanied by an increase in apoptosis, however, the rate of apoptosis was low as compared to free DOX. The involvement of both extrinsic and intrinsic pathways in this DOX induced apoptotic effect could be deciphered by the evaluation of caspase expression. In addition to this, significant morphological changes along with necrosis were also observed in DOX treated cells. When the results of $3 \mathrm{D}$ and $2 \mathrm{D}$ models were compared, significant differences were observed in proliferation indices and caspase levels.

In $4 \mathrm{~T} 1$ cells, mouse-derived progressive triple-negative breast cancer cells, Ki-67 analysis showed high proliferative activity of $80 \%$ which reduced to $27.2 \%$ after treatment with DOX. In case of $3 \mathrm{D}$ cultures for $4 \mathrm{~T} 1$ cells, the expression of caspase-3, 8 , and 9 increased by $20 \%, 40 \%$, and $20 \%$, respectively, as compared to the control cells. 2D cultures for MDA-MB-231 cells showed a reduction in
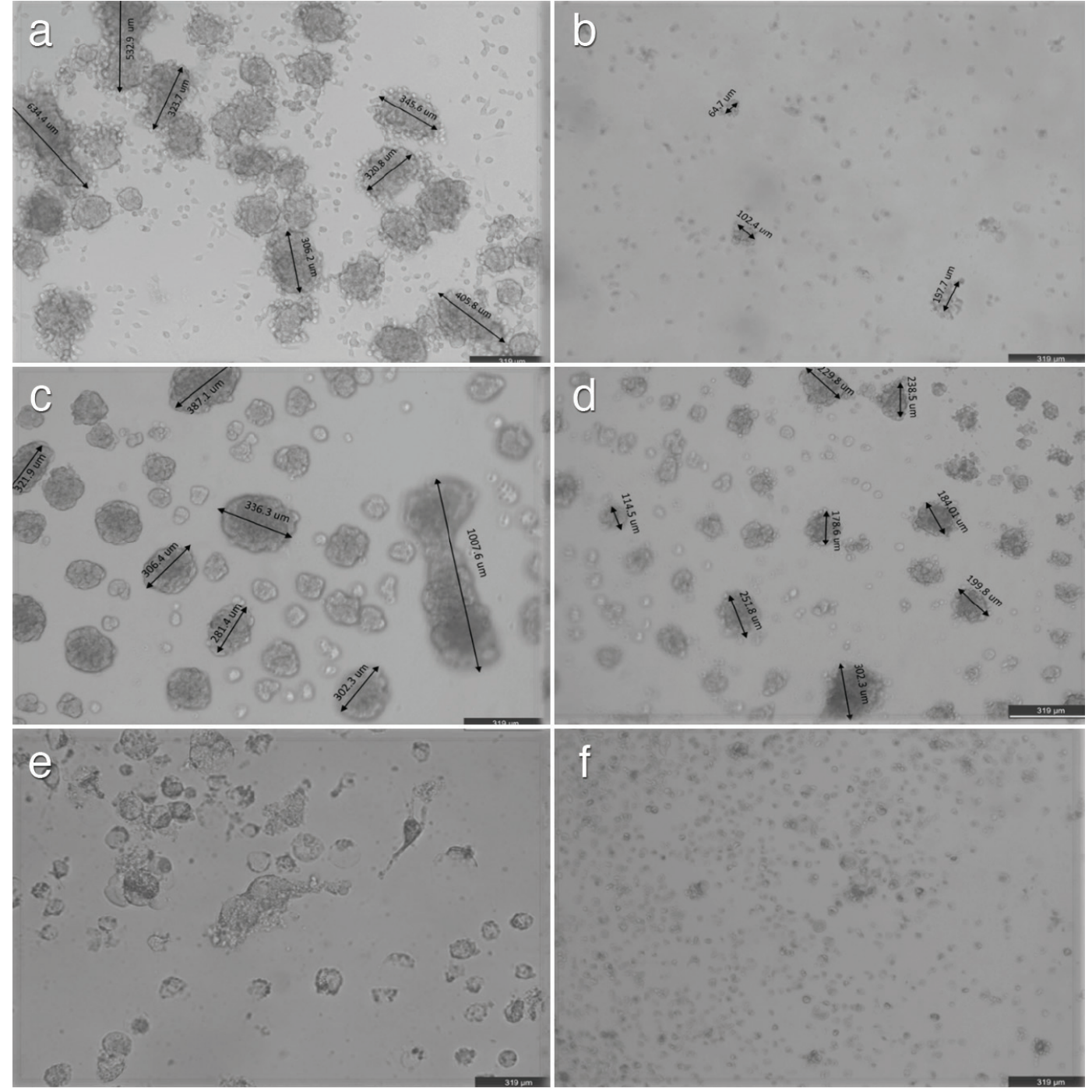

Figure 3. 3D Spherules in cell culture of a) Control 4T1 cells, b) DOX 4T1 cells; c) Control MCF7 cells, d) DOX MCF7 cells; e) control MDAMB231 cells and f) DOX MDAMB231 cells. Spherule number and diameters are prominently decreased in three cell lines after DOX incubation 
Ki-67 proliferation index from $98 \%$ to $13 \%$ upon treatment with DOX, while a significant increase was observed in the expression of caspase-3, 8, and 9. A comparative analysis for the three caspases showed maximum increase in case of caspase- 8 , indicating induction of both extrinsic and intrinsic pathways of apoptosis upon DOX treatment, but the activation of extrinsic pathway was comparatively higher.

In MDA-MB-231 cells, the activation of expression of caspase-3, 8, and 9 and reduction of proliferation index on DOX treatment was more significant as compared to $4 \mathrm{~T} 1$ cells. These two cell lines are characterized by the presence of triple-negative breast cancer features. However, they differ in terms of their origin, one belongs to humans while the other is derived from mice. In human-derived MDAMB-231 cells, DOX was found to be more effective in 2D cultures and its apoptosis-inducing effect was mediated via both extrinsic and intrinsic pathways. For MCF-7 cells, the increase in the expression of caspase-3, 8, and 9 expressions was lower as compared to 4T1 and MDA-MB-231 cells.

In $3 \mathrm{D}$ spheroid model for $4 \mathrm{~T} 1$ cell line, DOX treatment reduced Ki67 proliferation index by $40 \%$; however, the expression of caspase-3, 8 , and 9 was higher in the control group. Higher expression of caspases in the control group of 3D models might be attributed to lower support provided by the Matrigel, which could have induced cell death in spheroids after certain duration owing to the absence of suitable vascularization. Additionally, identification of negative cells in terms of caspase expression within the spheroids indicated absence of any issue in immunohistochemical staining. In order to identify the apoptosis mechanism induced by DOX treatment, expression of caspase-3, 8, and 9 was evaluated, which are indicative of involvement of common apoptotic, extrinsic, and intrinsic pathways, respectively. Similar to 2D model studies, caspase-3, 8, and 9 were activated in 3D model for all three cells. However, in case of $3 \mathrm{D}$ model studies, the control groups

Table 3. Flow Cytometry results for the effect of DOX and LipoDOX treatment for three cancer cell lines used in the study. Both DOX and LipoDOX induced cell death majorly via necrosis for all three cell lines $(p<0.05)$

\begin{tabular}{lcccc} 
\% & $\begin{array}{c}\text { Viability } \\
\text { AnnexinV-PI- }\end{array}$ & $\begin{array}{c}\text { Early apoptosis } \\
\text { AnnexinV + }\end{array}$ & $\begin{array}{c}\text { Late apoptosis } \\
\text { AnnexinV + PI + }\end{array}$ & $\begin{array}{c}\text { Necrosis } \\
\text { PI + }\end{array}$ \\
\hline 4T1 control & 61.6 & 0.2 & 14.9 & 23.3 \\
4T1 DOX & 0.2 & 0.0 & 0.6 & 1.2 \\
4T1 LipoDOX & 21 & 2 & 1.3 & 75.8 \\
MCF-7 control & 46.8 & 0.3 & 2.7 & 51.6 \\
MCF-7 DoX & 2.0 & 0.0 & 0.6 & 95.3 \\
MCF-7 LipoDOX & 17.2 & 1 & 5.6 & 81.2 \\
MDA-MB-231 control & 78 & 0.0 & 8.4 & 16.4 \\
MDA-MB-231DOX & 1.5 & 0.3 & 2.8 & 89.8 \\
MDA-MB-231 LipoDOX & 4.6 & 2.8 & 87.4
\end{tabular}

Annexin-V (-) and PI (-) cells are indicative of viability, Annexin-V (+) PI (-) cells represent early apoptotic cells, Annexin-V (+) and PI (+) cells indicate late apoptotic cells, and only PI (+) cells are necrotic

LipoDOX: Doxorubicin (DOX) entrapped in liposome; MB: Microbubbles

Table 4. TUNEL and Immunohistochemistry results for 3D cell culture of breast cancer cell lines. Proliferation index was measured in terms of Ki-67, which decreased upon treatment of the cells with DOX and LipoDOX ( $<<0.05)$ as compared to the control, for all three cell lines used in the study. Activation of both intrinsic and extrinsic pathways was involved in the induction of apoptosis

\begin{tabular}{|c|c|c|c|c|c|}
\hline$\%$ & TUNEL & Ki-67 & Caspase-3 & Caspase-8 & Caspase-9 \\
\hline 4T1 control & 3 & 80 & 60 & 50 & 30 \\
\hline 4T1 DOX & 35 & 27 & 80 & 90 & 50 \\
\hline 4T1 LipoDOX & 23 & 50 & 100 & 100 & 85 \\
\hline MCF-7 control & 5 & 80 & 30 & 49 & 20 \\
\hline MCF-7 DOX & 25 & 19 & 60 & 82 & 81 \\
\hline MCF-7 LipoDOX & 18 & 80 & 95 & 95 & 95 \\
\hline MDA-MB-231 control & 6 & 98 & 36 & 21 & 21 \\
\hline MDA-MB-231 DOX & 24 & 13 & 95 & 96 & 90 \\
\hline MDA-MB-231 LipoDOX & 15 & 50 & 100 & 73 & 70 \\
\hline
\end{tabular}


showed higher levels of expression for caspase-3, 8, and 9 as compared to $2 \mathrm{D}$ cultures. The treatment of MCF-7 cells with DOX resulted in $15 \%$ higher activation of caspase- 3,8 , and 9 as compared to the control cells in $3 \mathrm{D}$ model. In addition to this, a $20 \%$ reduction in proliferation index was observed post DOX treatment. For MDA-MB-231 cells, DOX treatment in $3 \mathrm{D}$ cell cultures resulted in a lower reduction in the proliferative index as compared to $2 \mathrm{D}$ model. In $3 \mathrm{D}$ model studies, the proliferation index for all three cell lines was observed to reduce after DOX treatment. This reduction in proliferation for $3 \mathrm{D}$ models was low as compared to the reduction observed in $2 \mathrm{D}$ cell culture models. Thus, all these results are indicative of the reduction in intracellular diffusion of DOX in 3D cell culture model, which further highlights the suitability of $3 \mathrm{D}$ cell cultures for drug efficacy studies.

\section{Discussion and Conclusion}

$2 \mathrm{D}$ cell culture models generally fail to mimic true in vivo tumor conditions $(17,18)$. In addition to this, 2D models generate very limited knowledge regarding the therapeutic efficacy of chemotherapeutic drugs. These limitations can be overcome by the use of $3 \mathrm{D}$ cell culture models. In comparison to 2D cultures, 3D cell culture models mimic in vivo tumor microenvironment in a much better way $(17,19)$. Several previous studies have reported significant differences in the results obtained for $2 \mathrm{D}$ and $3 \mathrm{D}$ cell culture models, particularly for the expression of various proteins and effects of chemotherapeutic drugs $(19,20)$. Similar results were obtained in the present study, where the expression of $\mathrm{Ki}-67$, caspase-3, caspase-8, and caspase- 9 in $2 \mathrm{D}$ cell cultures was comparatively low as compared to their $3 \mathrm{D}$ counterparts, irrespective of the cell line used for the evaluation.

In the present study, DOX and LipoDOX showed better 2D and 3D efficacy with increase in dose, for all the three cell lines studied. The observed change in efficacy was induced by the activation of apoptosis, particularly via induction of caspase- 8 . This was accompanied by a decrease in proliferative activity and viability in the $3 \mathrm{D}$ model, where significant reduction was observed in three-dimensional growth of the tumor cells. Moreover, the effective doses of LipoDOX required for the induction of cell proliferation inhibition and cell death were comparatively low as compared to free DOX, for all breast cancer cell lines used in the study. Thus, all these results suggest that lower doses of DOX would be safe for healthy cells, while ensuring effective inhibition of growth in breast cancer cells.

In the present study, the results obtained from the imaging studies highlighted the suitability of $3 \mathrm{D}$ cultures for breast cancer studies. In Matrigel matrix, the cells formed a structure similar to connective tissue around the spheroids. The occasional occurrence of apoptotic objects in the center of the spheroids and ductus structures was quite significant, especially in MCF-7 cells. These results were in agreement with a previous study, where establishment of spheroid like 3D model of breast cancer cell lines was associated with lower sensitivity toward chemotherapeutic drugs (18). These structures provided evidence for the suitability of the structures formed in 3D cultures for breast cancer studies.

In the present study, when spheroid structures were formed in $3 \mathrm{D}$ Matrigel model, DOX showed very limited diffusion into the cells, which was similar to in vivo conditions. Such a situation might be one of the responsible for the requirement of repeated administration of DOX at certain intervals under in vivo conditions. DOX showed antiproliferative and apoptosis-inducing effect in both $2 \mathrm{D}$ and $3 \mathrm{D}$ models for all three cell lines used in the study; however, this effect was quite variable among the three cell lines. These findings might preclinically reflect the difference in treatment responses observed in different individuals diagnosed with same cancer type. This further highlights the importance of personalized therapy for cancer treatment.

The present study was associated with certain limitations. In $3 \mathrm{D}$ models, great difficulty was witnessed while evaluating proliferation of spheroids using absorbance-based WST-1. Thus, trypan blue staining might be more effective and reliable for testing viability of spheroids in $3 \mathrm{D}$ cell culture models. Since DOX is characterized by absorbance maxima of $480 \mathrm{~nm}$, it might interfere with the absorbance-based measurements performed using WST-1.

In the present study, the effects of DOX and LipoDOX treatment on three different breast cancer cell lines were investigated in $2 \mathrm{D}$ and $3 \mathrm{D}$ cell culture models. Both drug formulations were found to be effective in all three breast cancer cells tested. The currently used strategies mainly focus on utilizing tumor targeting drug delivery vehicles to ensure local delivery of the drugs to the tissue of interest. Such targeted delivery minimizes the side effects and improves the antitumoral effect of the drug. In the present study, DOX-loaded MBs were targeted to the tumor cells by Epidermal growth factor receptor (EGFR); however, in vitro flow experiments could not be performed owing to the buoyancy of the MBs. The results for the antitumoral effects of DOX and LipoDOX obtained in the present study have laid down the foundation of a future study that will focus on the development of liposomal DOX-MB complex targeted specifically to breast cancer using EGFR. This study will also evaluate the local delivery of DOX in mouse model both in the presence and absence of ultrasound. Thus, the present study provided a suitable method for the evaluation of anti-proliferative and anti-apoptotic effects of liposomal doxorubicin in $3 \mathrm{D}$ cell culture models with different types of breast cancer cells.

\section{Acknowledgements}

This study was supported by Dokuz Eylül University Scientific Research Commission with project number 2018.KB.SAG.033. The flow experiments and $\mathrm{MB}$ production stages of the project were completed within the scope of the Scientific and Technological Research Council of Turkey: TUBITAK (project no: 213M668).

Ethics Committee Approval: This study was approved by Dokuz Eylül University, Noninvasive Ethics Committee (date: 09.11.2020, no: 2017/2622).

Informed Consent: This study is done on cell lines so that it does not require consent form

Peer-review: Externally peer-reviewed.

\section{Author Contributions}

Conception: M.A., S.A.; Design: S.A., S.K.; Supervision: S.A., E.O., S.K.; Resources: M.A., S.A., E.O., Z.A., S.K.; Data Collection and/or Processing: M.A, S.A., S.K.; Analysis and/or Interpretation: M.A., S.A., S.K.; Literature Search: M.A., S.A., Z.A., S.K..; Writing Manuscript: M.A., S.A., Z.A., S.K.; Critical Review: S.A., S.K.O, E.O.

Conflict of Interest: The authors have no conflicts of interest to declare.

Financial Disclosure: This study was supported by Dokuz Eylül University Scientific Research Commission with project number: 2018.KB.SAG.033. The flow experiments and $\mathrm{MB}$ production stages of the project were completed within the scope of TÜBİTAK (project no: 213M668). 


\section{References}

1. Mahvi DA, Liu R, Grinstaff MW, Colson YL, Raut CP. Local cancer recurrence: the realities, challenges, and opportunities for new therapies. CA Cancer J Clin 2018; 68: 488-505. (PMID: 30328620) [Crossref]

2. Narod SA. Personalised medicine and population health: breast and ovarian cancer. Hum Genet 2018; 137: 769-778. (PMID: 30328515) [Crossref]

3. Maffini MV, Soto AM, Calabro JM, Ucci AA, Sonnenschein C. The stroma as a crucial target in rat mammary gland carcinogenesis. J Cell Sci 2004; 117: 1495-1502. (PMID: 14996910) [Crossref]

4. Smalley M, Piggott L, Clarkson R. Breast cancer stem cells: obstacles to therapy. Cancer Lett 2013; 338: 57-62. (PMID: 22554712) [Crossref]

5. Cochran MC, Eisenbrey J, Ouma RO, Soulen M, Wheatley MA. Doxorubicin and paclitaxel loaded microbubbles for ultrasound triggered drug delivery. Int J Pharm 2011; 414: 161-170. (PMID: 21609756) [Crossref]

6. Yan F, Li L, Deng Z, Jin Q, Chen J, Yang W, et al. Paclitaxel-liposomemicrobubble complexes as ultrasound-triggered therapeutic drug delivery carriers. J Control Release 2013; 166: 246-255. (PMID: 23306023) [Crossref]

7. Vail DM, Amantea MA, Colbern GT, Martin FJ, Hilger RA, Working PK. Pegylated liposomal doxorubicin: proof of principle using preclinical animal models and pharmacokinetic studies. Semin Oncol 2004; 31: 1635. (PMID: 15717736) [Crossref]

8. Harris L, Batist G, Belt R, Rovira D, Navari R, Azarnia N, et al. Liposomeencapsulated doxorubicin compared with conventional doxorubicin in a randomized multicenter trial as first-line therapy of metastatic breast carcinoma. Cancer 2002: 94: 25-36. (PMID: 11815957) [Crossref]

9. Bush N, Healey A, Shah A, Box G, Kirkin V, Eccles S, et al. Theranostic attributes of acoustic cluster therapy and its use for enhancing the effectiveness of liposomal doxorubicin treatment of human triple negative breast cancer in mice. Front Pharmacol 2020; 11: 75. (PMID: 32153400) [Crossref]

10. Pothuri B, Brodsky AL, Sparano JA, Blank SV, Kim M, Hershman DL, et al. Phase I and pharmacokinetic study of veliparib, a PARP inhibitor, and pegylated liposomal doxorubicin (PLD) in recurrent gynecologic cancer and triple negative breast cancer with long-term follow-up. Cancer Chemother Pharmacol 2020; 85: 741-751. (PMID: 32055930) [Crossref]
11. Zhao T, Zhou H, Lei L, Guo C, Yang Q, Gong T, et al. A new tandem peptide modified liposomal doxorubicin for tumor "ecological therapy". Nanoscale 2020; 12: 3359-3369. (PMID: 31984408) [Crossref]

12. Navashenaq JG, Zamani P, Nikpoor AR, Tavakkol-Afshari J, Jaafari MR. Doxil chemotherapy plus liposomal P5 immunotherapy decreased myeloid-derived suppressor cells in murine model of breast cancer. Nanomedicine 2020; 24: 102150. doi: 10.1016/j.nano.2020.102150 (PMID: 31931230) [Crossref]

13. Cao D, Zhang X, Akabar MD, Luo Y, Wu H, Ke X, et al. Liposomal doxorubicin loaded PLGA-PEG-PLGA based thermogel for sustained local drug delivery for the treatment of breast cancer. Artif Cells Nanomed Biotechnol 2019; 47: 181-191. (PMID: 30686051) [Crossref]

14. Lin CY, Li JR, Tseng HC. Enhancement of focused ultrasound with microbubbles on the treatments of anticancer nanodrug in mouse tumors. Nanomedicine 2012; 8: 900-907. (PMID: 22033084) [Crossref]

15. Tinkov S, Coester C, Serba S, Geis NA, Katus HA, Winter G, et al. New doxorubicin-loaded phospholipid microbubbles for targeted tumor therapy: in-vivo characterization. J Control Release 2010; 148: 368-372. (PMID: 20868711) [Crossref]

16. Onercan C. Development of ultrasound triggered drug delivery systems for cancer treatment. Izmir Institute of Technology, Master Thesis, Turkey, 2019. [Crossref]

17. Weigelt B, Ghajar CM, Bissell MJ. The need for complex 3D culture models to unravel novel pathways and identify accurate biomarkers in breast cancer. Adv Drug Deliv Rev 2014; 69-70: 42-51. (PMID: 24412474) [Crossref]

18. Imamura $Y$, Mukohara $T$, Shimono $Y$, Funakoshi $Y$, Chayahara N, Toyoda $\mathrm{M}$, et al. Comparison of $2 \mathrm{D}$ - and $3 \mathrm{D}$-culture models as drug-testing platforms in breast cancer. Oncol Rep 2015; 33: 1837-1843. (PMID: 25634491) [Crossref]

19. Lee JM, Mhawech-Fauceglia P, Lee N, Parsanian LC, Lin YG, Gayther $\mathrm{SA}$, et al. A three-dimensional microenvironment alters protein expression and chemosensitivity of epithelial ovarian cancer cells in vitro. Lab Invest 2013; 93: 528-542. (PMID: 23459371) [Crossref]

20. Vinci M, Gowan S, Boxall F, Patterson L, Zimmermann M, Court W, et al. Advances in establishment and analysis of three-dimensional tumor spheroid-based functional assays for target validation and drug evaluation. BMC Biol 2012; 10: 29. (PMID: 22439642) [Crossref] 